\title{
Effects of entanglement and instanton suppression at finite temperature in a SU(2) EPNJL model with anomaly
}

\author{
M. C. Ruivo $*$ P. Costa $\rfloor^{\dagger}$ and C. A. de Sousat \\ Centro de Física Computacional, Department of Physics, \\ University of Coimbra, P-3004 - 516 Coimbra, Portugal
}

(Dated: June 30, 2018)

\begin{abstract}
We investigate the phase transitions characterized by deconfinement and restoration of chiral and axial symmetries, at finite temperature, in the framework of QCD inspired models. We compare the results obtained in the SU(2) Polyakov-Nambu-Jona-Lasinio model with anomaly and in its extended version, the Entangled Polyakov-Nambu-Jona-Lasinio model. In the last version, four-quark vertices with entanglement between the chiral condensate and the Polyakov loop are considered. The thermodynamics of the phase transitions, the meson spectrum, and in particular the convergence of axial and chiral partners, will be analyzed, as well as the topological susceptibility. We find that an explicit temperature dependence of the coupling vertices is necessary in both models in order to have effective restoration of the $\mathrm{U}_{A}(1)$ symmetry.

PACS numbers: 11.30.Rd, 11.55.Fv, 11.10.Wx
\end{abstract}

\footnotetext{
* Corresponding author: M. C. Ruivo; maria@teor.fis.uc.pt

$\dagger$ pcosta@teor.fis.uc.pt

$\ddagger$ celia@teor.fis.uc.pt
} 


\section{INTRODUCTION}

The study of matter under extreme conditions of temperatures and/or densities is currently an important field of research in physics both experimentally and theoretically. In this limit, quantum chromodynamics (QCD), the theory of strong interactions, predicts that matter becomes a plasma of deconfined quarks and gluons. In spite of the success of many aspects of the strong interaction physics, there are important features to clarify, such as the behavior of the masses of the mesons and the thermodynamics of strongly interacting matter in the region of the transition from hadronic to quark matter.

Monte Carlo simulations in lattice gauge theory provide a powerful nonperturbative first principle approach to QCD, but the region of large densities and low temperatures essentially remains inaccessible to lattice simulations. This encourages the study and enlargement of effective models with QCD symmetries such as Polyakov-Nambu-Jona-Lasinio (PNJL) model. The PNJL model is an effective model which respects important symmetries of QCD action. It contains quarks as fundamental degrees of freedom allowing for a self-consistent description of chiral symmetry breaking, a key feature of QCD in its low temperature and density regime. Besides, the coupling to the Polyakov loop allows one to describe the (statistical) confinement/deconfinement phase transition by taking into account a static gluonic field in which quarks propagate [1 4]. The NJL part of the model (where a pointlike interaction mimicking frozen gluon is introduced) can describe the chiral phase transition and its associate order parameter, the quark condensate. On the other hand, it is known that in pure gauge theory there is a confined/deconfined phase transition whose associate parameter is the Polyakov loop. Quarks couple simultaneously to the chiral condensate and to the Polyakov loop, allowing one to examine the correlation between confinement and chiral symmetry breaking whose direct relation is not yet shown in QCD.

The PNJL model has proven to be successful in reproducing lattice data concerning QCD thermodynamics [3]: the coupling to the Polyakov loop, resulting in a statistical suppression of the unwanted quark contributions to the thermodynamics below the critical temperature, allows a good description compatible with lattice results. It has been also used to describe the full phase diagram, essentially at the mean field level.

An important query on QCD thermodynamics is the proximity or coincidence of the two phase transitions characterized respectively by restoration of chiral symmetry and decon- 
finement. Lattice QCD results concerning this subject have been a matter of debate and, while for $N_{f}=2+1$ flavors these two phase transitions are reported to take place at distinct temperatures [5], for $N_{f}=2$ flavors results indicate that the two phase transitions occur at the same temperature [6], $T_{C}=174(3)(6) \mathrm{MeV}$. This effect could be the result of strong correlations (entanglement) between the quark condensate and the Polyakov field, $\Phi$. An extension of the PNJL model has been proposed where this entanglement is taken into account, by endowing the four-quark interaction of the model with a dependence on the Polyakov field. This is the so-called entangled Polyakov-Nambu-Jona-Lasinio (EPNJL) [7, 8]. In this model the two phase transitions occur at the same temperature and it is possible to reproduce lattice values.

A longstanding question is whether the $\mathrm{U}_{A}(1)$ symmetry, that is broken in the vacuum at the quantum level by instantons, is still broken in the chiral symmetric phase. If the amount of the $\mathrm{U}_{A}(1)$ symmetry breaking decreases with temperature, the question is what fraction of it remains above the critical temperature and whether and when this symmetry is restored. It has been pointed out that there are phenomenological consequences for the nature of the phase transition depending on the degree of anomaly present at the critical temperature [9, 10]. Several observables can exhibit signals of the restoration of axial symmetry, like the topological susceptibility, the meson axial chiral partners, and the $\eta^{\prime}$ mass. Lattice calculations have found evidence of the decrease of the topological susceptibility with temperature in former works [11] as well as in recent ones [9, 10, 12]. In the last works attention has also been given to the meson correlators of chiral and axial partners, which should become degenerate when both symmetries are restored. Concerning the $\eta^{\prime}$, the decrease of its mass in medium has been predicted in several theoretical works [13 16], and recent experimental results [17] in $\mathrm{Au}+\mathrm{Au}$ collisions are compatible with a decrease of about $200 \mathrm{MeV}$, which could indicate the return of the 9th "prodigal" Goldstone boson.

QCD inspired models have also been used to study the restoration of axial symmetry, in particular the NJL model [15, 18] and its extended version, the PNJL model [19, 20]. A procedure used in several works to account for the decrease of the topological susceptibility is to allow a temperature dependence of the anomaly coefficient [13, 15, 18]. In previous works, we have discussed the possible relation between the restorations of chiral and axial symmetries, both in SU(3) NJL model and PNJL models including the 't Hooft interaction [21]. It has been found that, by allowing the presence of high momentum quarks at finite 
temperature (using an infinite cutoff as explained in [21]) observables related with the $\mathrm{U}_{A}(1)$ symmetry breaking vanish (the topological susceptibility as well as the mass difference between the meson axial partners) as a natural consequence of the effective restoration of chiral symmetry, without the need of additional assumptions. However, the situation is different in the framework of the SU(2) NJL and PNJL models, as we have shown in a recent work [22]. In this case, an additional mechanism that ensures the suppression of instantons with temperature is needed in order that the meson axial partners become degenerate. This was achieved by taking the anomaly coefficient as a decreasing function of the temperature. If such a mechanism is not considered, and although the meson chiral partners degenerate and the topological susceptibility vanishes, signals of the anomaly will remain long after the critical temperature since the meson axial partners do not converge.

The goal of the present paper, besides enlarging the previous study exploring different schemes of temperature dependence of the coupling coefficients, is to extend it to the $\mathrm{SU}(2)$ EPNJL model. A comparative study will be performed and the question whether there is any relation between the deconfinement, restoration of chiral and axial symmetries will be analyzed. Since restoration of axial symmetry in the PNJL model can only be achieved by assuming a temperature dependence of coupling coefficients, we will discuss whether an explicit temperature dependence of the vertices is also necessary in the EPNJL model for this purpose or if the temperature dependence through the Polyakov field is sufficient.

The relevant orderlike parameters will be analyzed and special attention will be paid to the phase structure underlying the confinement-deconfinement and chiral transitions, as well as to thermodynamic quantities, such as the pressure, the behavior of the topological susceptibility and the convergence of meson axial and chiral partners. Section II is devoted to the description of the models, Sec. III to the discussion of the results and, finally, we summarize our findings in Sec. IV.

\section{MODEL AND FORMALISM}

We will use the SU(2) PNJL model with a 't Hooft interaction simulating the $\mathrm{U}_{A}(1)$ anomaly (instanton effects). Afterwards, several mechanisms of temperature dependence of the coupling coefficients are included, in particular the dependence of temperature through the Polyakov loop field, leading to entanglement of deconfinement and restoration of chiral 
symmetry (EPNJL model).

The PNJL Lagrangian with explicit chiral symmetry breaking where the quarks couple to a (spatially constant) temporal background gauge field (represented in terms of Polyakov loops) is given by [3, 23]

$$
\mathcal{L}_{P N J L}=\bar{q}\left(i \gamma^{\mu} D_{\mu}-\hat{m}\right) q+\mathcal{L}_{1}+\mathcal{L}_{2}-\mathcal{U}(\Phi[A], \bar{\Phi}[A] ; T)
$$

with two different interacting parts

$$
\begin{aligned}
& \mathcal{L}_{1}=g_{1}\left[(\bar{q} q)^{2}+\left(\bar{q} i \gamma_{5} \vec{\tau} q\right)^{2}+(\bar{q} \vec{\tau} q)^{2}+\left(\bar{q} i \gamma_{5} q\right)^{2}\right] \\
& \mathcal{L}_{2}=g_{2}\left[(\bar{q} q)^{2}+\left(\bar{q} i \gamma_{5} \vec{\tau} q\right)^{2}-(\bar{q} \vec{\tau} q)^{2}-\left(\bar{q} i \gamma_{5} q\right)^{2}\right] .
\end{aligned}
$$

The quark fields $q=(u, d)$ are defined in Dirac and color fields, respectively with two flavors, $N_{f}=2$ and three colors, $N_{c}=3$, the coupling coefficients $g_{1}$ and $g_{2}$ have dimension energy ${ }^{-2}$, and $\hat{m}=\operatorname{diag}\left(m_{u}, m_{d}\right)$ is the current quark mass matrix. For simplicity we assume below that $m_{u}=m_{d}=m$. The original NJL model is reproduced for $g_{1}=g_{2}$.

The Lagrangian (1) is chiral invariant in the limit where the current quark masses vanish. Both terms $\mathcal{L}_{1}$ and $\mathcal{L}_{2}$ are invariant upon $\mathrm{SU}(2)_{L} \otimes \mathrm{SU}(2)_{R} \otimes \mathrm{U}(1)$-type transformations, but the $\mathcal{L}_{2}$ component makes the Lagrangian noncovariant upon $\mathrm{U}_{A}(1)$ transformations. The $\mathcal{L}_{2}$ term, which can be explicitly written in the form of a determinant (see [22]) and identified as an interaction induced by instantons, according to 't Hooft, explicitly breaks the axial symmetry even in the chiral limit.

The quarks are coupled to the gauge sector via the covariant derivative $D^{\mu}=\partial^{\mu}-i A^{\mu}$. The strong coupling constant $g_{\text {Strong }}$ has been absorbed in the definition of $A^{\mu}: A^{\mu}(x)=$ $g_{\text {Strong }} \mathcal{A}_{a}^{\mu}(x) \frac{\lambda_{a}}{2}$, where $\mathcal{A}_{a}^{\mu}$ is the $\mathrm{SU}_{c}(3)$ gauge field and $\lambda_{a}$ are the Gell-Mann matrices. Besides, in the Polyakov gauge and at finite temperature $A^{\mu}=\delta_{0}^{\mu} A^{0}=-i \delta_{4}^{\mu} A^{4}$.

The Polyakov loop $\Phi$ (the order parameter of $\mathbb{Z}_{3}$ symmetric/broken phase transition in pure gauge) is the trace of the Polyakov line defined by $\Phi=\frac{1}{N_{c}}\left\langle\left\langle\mathcal{P} \exp i \int_{0}^{\beta} d \tau A_{4}(\vec{x}, \tau)\right\rangle\right\rangle_{\beta}$.

The pure gauge sector is described by an effective potential $\mathcal{U}(\Phi[A], \bar{\Phi}[A] ; T)$ chosen to reproduce at the mean-field level the results obtained in lattice calculations:

$$
\frac{\mathcal{U}(\Phi, \bar{\Phi} ; T)}{T^{4}}=-\frac{a(T)}{2} \bar{\Phi} \Phi+b(T) \ln \left[1-6 \bar{\Phi} \Phi+4\left(\bar{\Phi}^{3}+\Phi^{3}\right)-3(\bar{\Phi} \Phi)^{2}\right]
$$

where

$$
a(T)=a_{0}+a_{1}\left(\frac{T_{0}}{T}\right)+a_{2}\left(\frac{T_{0}}{T}\right)^{2} \text { and } b(T)=b_{3}\left(\frac{T_{0}}{T}\right)^{3}
$$




\begin{tabular}{cccc}
\hline$a_{0}$ & $a_{1}$ & $a_{2}$ & $b_{3}$ \\
\hline 3.51 & -2.47 & 15.2 & -1.75 \\
\hline
\end{tabular}

TABLE I. Parameters for the effective potential in the pure gauge sector.

The effective potential exhibits the feature of a phase transition from color confinement $\left(T<T_{0}\right.$, the minimum of the effective potential being at $\Phi=0$ ) to color deconfinement $\left(T>T_{0}\right.$, the minima of the effective potential occurring at $\left.\Phi \neq 0\right)$.

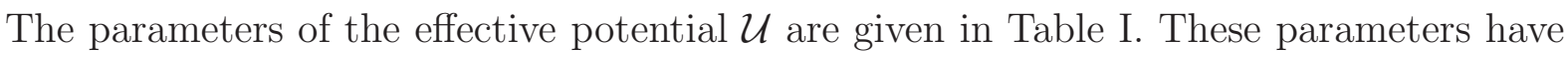
been fixed in order to reproduce the lattice data for the expectation value of the Polyakov loop and QCD thermodynamics in the pure gauge sector [24, 25].

The parameter $T_{0}$ is the critical temperature for the deconfinement phase transition within a pure gauge approach: it was fixed to $270 \mathrm{MeV}$, according to lattice findings. This choice ensures an almost exact coincidence between chiral crossover and deconfinement at finite temperature, as observed in lattice calculations.

The Lagrangian density (11) can be rewritten as

$$
\begin{aligned}
\mathcal{L}_{P N J L} & =\bar{q}\left(i \gamma^{\mu} D_{\mu}-\hat{m}\right) q+G_{s}\left[(\bar{q} q)^{2}+\left(\bar{q} i \gamma_{5} \vec{\tau} q\right)^{2}\right]+G_{a}\left[(\bar{q} \vec{\tau} q)^{2}+\left(\bar{q} i \gamma_{5} q\right)^{2}\right] \\
& -\mathcal{U}(\Phi[A], \bar{\Phi}[A] ; T)
\end{aligned}
$$

where, as quoted below, $G_{s}=g_{1}+g_{2}$ is related to the $\pi$ and $\sigma$ mesons and $G_{a}=g_{1}-g_{2}$ to $\eta$ and $a_{0}$ mesons.

The PNJL grand canonical potential density in the $\mathrm{SU}_{f}(2)$ sector can be written as [3, 26]

$$
\begin{aligned}
\Omega(\Phi, \bar{\Phi}, M ; T, \mu) & =\mathcal{U}(\Phi, \bar{\Phi}, T)+4 G_{s} N_{f}\left\langle\bar{q}_{i} q_{i}\right\rangle^{2}-2 N_{c} N_{f} \int_{\Lambda} \frac{\mathrm{d}^{3} p}{(2 \pi) 3} E_{i} \\
& -2 N_{f} T \int \frac{\mathrm{d}^{3} p}{(2 \pi)^{3}}\left(z_{\Phi}^{+}\left(E_{i}\right)+z_{\Phi}^{-}\left(E_{i}\right)\right),
\end{aligned}
$$

where $E_{i}$ is the quasiparticle energy for the quark $i: E_{i}=\sqrt{\mathbf{p}^{2}+M_{i}^{2}}$, and $z_{\Phi}^{+}$and $z_{\Phi}^{-}$are the partition function densities. 
The explicit expression of $z_{\Phi}^{+}$and $z_{\Phi}^{-}$are given by:

$$
\begin{aligned}
& z_{\Phi}^{+}\left(E_{i}\right) \equiv \operatorname{Tr}_{c} \ln \left[1+L^{\dagger} e^{-\beta\left(E_{i}+\mu\right)}\right]=\ln \left\{1+3\left(\bar{\Phi}+\Phi e^{-\beta\left(E_{i}+\mu\right)}\right) e^{-\beta\left(E_{i}+\mu\right)}+e^{-3 \beta\left(E_{i}+\mu\right)}\right\} \\
& z_{\Phi}^{-}\left(E_{i}\right) \equiv \operatorname{Tr}_{c} \ln \left[1+L e^{-\beta\left(E_{i}-\mu\right)}\right]=\ln \left\{1+3\left(\Phi+\bar{\Phi} e^{-\beta\left(E_{i}-\mu\right)}\right) e^{-\beta\left(E_{i}-\mu\right)}+e^{-3 \beta\left(E_{i}-\mu\right)}\right\}
\end{aligned}
$$

A word is in order to describe the role of the Polyakov loop in the present model. Almost all physical consequences of the coupling of quarks to the background gauge field stem from the fact that in the expression of $z_{\Phi}, \Phi$ or $\bar{\Phi}$ appear only as a factor of the one- or twoquarks (or antiquarks) Boltzmann factor, for example $e^{-\beta\left(E_{i}-\mu\right)}$ and $e^{-2 \beta\left(E_{i}-\mu\right)}$. Hence, when $\Phi, \bar{\Phi} \rightarrow 0$ (signaling what we designate as the "confined phase") only $e^{-3 \beta\left(E_{i}-\mu\right)}$ remains in the expression of the grand canonical potential, leading to a thermal bath with a small quark density. On the contrary, $\Phi, \bar{\Phi} \rightarrow 1$ (in the "deconfined phase") gives a thermal bath with all 1-, 2- and 3-particle contributions and a significant quark density [26, 27].

This formalism, presented here for completeness in the grand canonical approach, will be employed in the present work with $\mu=0$. This condition implies $\Phi=\bar{\Phi}$.

We can redefine the coupling constants such as the set $\left(g_{1}, g_{2}\right)$ or $\left(G_{s}, G_{a}\right)$ will be replaced by $(G, c)$ in the following parametrization:

$$
G_{s}=g_{1}+g_{2}=G, \quad G_{a}=g_{1}-g_{2}=G(1-2 c),
$$

where $c \in\{0,1\}[28,29]$ is a parameter that now specifies the degree of $\mathrm{U}_{A}(1)$ symmetry breaking. In the present work we take $c=0.2$, a value which gives an adequate degree of anomaly in the vacuum [22], as will be shown in the sequel by the choice of model parameters. Notice that $g_{1}=G(1-c)$ is the coupling constant of the four-quark vertex associated with chiral symmetry effects, while $g_{2}=c G$ is the anomaly coefficient and is, for the present choice, $25 \%$ of $g_{1}$.

As it is well known, in pure gauge theory, the Polyakov potential induces a first-order phase transition at $T=T_{0}$. The PNJL model with the original $T_{0}=270 \mathrm{MeV}$, that reproduces pure gauge lattice QCD data, yields to a small difference between chiral and deconfinement transition temperatures. This value is however significant when we rescale $T_{0}$ to $210 \mathrm{MeV}$, derived by renormalization-group considerations [30] so as to reproduce the lattice $\mathrm{QCD}$ result, $T_{d}=177 \mathrm{MeV}$ for the deconfinement transition temperature as will be 
seen in the next section. Consequently, the PNJL result is not consistent with lattice QCD data for the transition temperatures. This indicates that the entanglement between chiral and deconfinement transitions is weak in this model. Following the suggestions of other authors [7, 8], we implement an explicit dependence of $G$ on $\Phi$ assuming the following form:

$$
G(\Phi)=G\left[1-\alpha_{1} \Phi \bar{\Phi}-\alpha_{2}\left(\Phi^{3}+\bar{\Phi}^{3}\right)\right]
$$

which respect chiral, $P, C$ and the extended $\mathbb{Z}_{3}$ symmetries. We use the parameters of reference Ref. [7], where they have been fixed to reproduce the available lattice QCD data [6, 31]. This leads to the values $\alpha_{1}=\alpha_{2}=0.2$ and $T_{0}=170 \mathrm{MeV}$.

A standard calculation leads straightforwardly to the gap equation and to the meson propagators. The following gap equations are obtained:

$$
M_{i}=m_{i}-4 G\langle\bar{q} q\rangle_{i},
$$

where one identifies $i=u, d$ and $M_{i}$ as the constituent quark mass. The quark condensates are determined by

$$
\langle\bar{q} q\rangle_{i}=-i \operatorname{Tr} \frac{1}{\hat{p}-M_{i}}=-i \operatorname{Tr} S_{i}(p),
$$

being $S_{i}(p)=\left(\not p-M_{i}+i \varepsilon\right)^{-1}$ the propagator of quarks.

The mass spectra of the mesons is obtained by the analysis of the pole structure of the meson propagator, given by

$$
1-4 G_{s, a} \Pi_{\mathcal{M}}\left(q^{2}=M_{\mathcal{M}}^{2}\right)=0
$$

where

$$
\Pi_{\mathcal{M}}\left(q^{2}\right)=i \int \frac{d^{4} p}{(2 \pi)^{4}} \operatorname{Tr}\left[\mathcal{O}_{\mathcal{M}} S(p+q) \mathcal{O}_{\mathcal{M}} S(p)\right]
$$

is the polarization operator for the quark-antiquark system regarding the channel with quantum numbers $\{\mathcal{M}\}$ in the mesonic sector. As mentioned above, $G_{s}$ is related to $\pi$ and $\sigma$ mesons and $G_{a}$ to $\eta$ and $a_{0}$ mesons.

The topological susceptibility, $\chi$, is an essential parameter for the study of the problem of breaking and restoration of the $\mathrm{U}_{A}(1)$ symmetry. The topological susceptibility is defined as:

$$
\chi=\int \mathrm{d}^{4} x\langle 0|T Q(x) Q(0)| 0\rangle_{\mathrm{c}}
$$




\begin{tabular}{||c||c|c|c|c|c|c|c||}
\hline \hline & $\begin{array}{c}f_{\pi} \\
{[\mathrm{MeV}]}\end{array}$ & $\begin{array}{c}\langle\bar{q} q\rangle^{1 / 3} \\
{[\mathrm{MeV}]}\end{array}$ & $\begin{array}{c}m_{\pi} \\
{[\mathrm{MeV}]}\end{array}$ & $\begin{array}{c}m_{\sigma} \\
{[\mathrm{MeV}]}\end{array}$ & $\begin{array}{c}m_{\eta} \\
{[\mathrm{MeV}]}\end{array}$ & $\begin{array}{c}m_{a_{0}} \\
{[\mathrm{MeV}]}\end{array}$ & $\begin{array}{c}\chi^{1 / 4} \\
{[\mathrm{MeV}]}\end{array}$ \\
\hline \hline Model & 93 & -241 & 140.2 & 803.7 & 704.5 & 919.8 & 180.8 \\
\hline Experimental /Lattice & 92.4 & -267 & 135.0 & $400-1200$ & 547.3 & 984.7 & 180 \\
\hline \hline
\end{tabular}

TABLE II. Numerical values for the calculated observables compared with experimental and lattice results, obtained with $\Lambda=590 \mathrm{MeV}, G_{s} \Lambda^{2}=G \Lambda^{2}=2.435, c=0.2$, and $m=6 \mathrm{MeV}$.

where $c$ means connected diagrams, $T$ the time order operator and $Q(x)$ is of the form

$$
Q(x)=2 g_{2}\left[\operatorname { d e t } \left[\left(\bar{q}\left(1-\gamma_{5}\right) q-\operatorname{det}\left[\left(\bar{q}\left(1+\gamma_{5}\right) q\right]\right. \text {. }\right.\right.\right.
$$

Taking only into account the connected diagrams, and only the terms of order $1 / N_{c}$, following a similar approach to [18], we arrive at the following expression for the topological susceptibility (see [22] for details):

$$
\chi=4 N_{f} g_{2}^{2}\langle\bar{q} q\rangle^{2} \frac{4 I_{1}}{1-16 G_{a} I_{1}}
$$

where $I_{1}=-\langle\bar{q} q\rangle_{i} / 4 M_{i}$.

The present PNJL model has four parameters in the NJL sector: $m, \Lambda, g_{1}$, and $g_{2}$. We choose to adjust the parameters in vacuum by fitting to well-known experimental data or lattice values: the mass of the pion, its decay constant, the quark condensate and the topological susceptibility that are shown in Table II The masses of the $\sigma, \eta$, and $a_{0}$ mesons come as outputs. This set of parameters is crucial to get the correct description of isentropic trajectories in the $T \rightarrow 0$ limit [32].

\section{RESULTS AND DISCUSSION}

In a previous work [22], from the analysis of the behavior of the the topological susceptibility and of meson axial partners, we concluded that effective restoration of axial symmetry could only be achieved when $g_{2}$ was taken as a decreasing function of temperature. By using $g_{2}(T)=g_{2}(0) /\left(1+\exp \left(\left(T-T_{0}\right) / 10\right)\right)$ we got the full restoration of axial symmetry even with a finite cutoff, $\Lambda$, at finite temperature; this ansatz is interpreted as an explicit mechanism of instanton suppression. In the present work, we will enlarge the investigation 


\begin{tabular}{||c||c|c|c||}
\hline \hline & I & II & III \\
& & $($ Scenario $A)$ & $($ Scenario B $)$ \\
\hline PNJL & $g_{1}, g_{2}$ & $g_{1}, g_{2}(T)$ & $g_{1}(T), g_{2}(T)$ \\
\hline EPNJL & $g_{1}(\Phi), g_{2}(\Phi)$ & $g_{1}(\Phi), g_{2}(\Phi, T)$ & $g_{1}(\Phi, T), g_{2}(\Phi, T)$ \\
\hline \hline
\end{tabular}

TABLE III. Scenarios of the temperature dependence of $g_{1}$ and $g_{2}$ in PNJL and EPNJL models: in column I there is no explicit dependence on the temperature, columns II and III correspond to the scenarios $\mathrm{A}$ and $\mathrm{B}$.

of possible temperature dependence of the coupling coefficients and its consequences for several observables. We consider two scenarios, that in the PNJL model are (see Table III)

- Scenario $A$ - We will keep $g_{1}$ and $g_{2}$ as independent parameters. At finite temperature we may allow $g_{2}$ to have an explicit dependence on temperature but $g_{1}$ is kept constant.

- Scenario $B$ - We will use the redefinition of Eq. (10) allowing for

$$
g_{1}=G(1-c), \quad g_{2}=G c
$$

In the last scenario $g_{1}$ and $g_{2}$ are not independent, but $G_{s}=G$ will be kept always constant; on the contrary, $G_{a}$ varies since

$$
c(T)=0.2 f(T), \text { where } f(T)=1 /\left(1+\exp \left(\left(T-T_{0}\right) / 10\right)\right)
$$

In this case only the topological susceptibility and the $\eta$ and $a_{0}$ meson masses will be affected. The other quantities have the same behavior as when $g_{1}$ and $g_{2}$ are kept constant.

In the EPNJL we have equivalent scenarios with the replacement: $g_{1} \rightarrow g_{1}(\Phi)$ and $g_{2} \rightarrow g_{2}(\Phi)$. In both cases we allow $T_{0}$ (the critical temperature for the deconfinement phase transition within a pure gauge approach, usually fixed to $270 \mathrm{MeV}$ ) to have several values and we discuss this effect. We will compare results obtained in the framework of the PNJL and EPNJL models for the characteristic temperatures, the pressure, the topological susceptibility, and the masses of chiral and axial meson partners. Along the work, we will always consider the cutoff $\Lambda \rightarrow \infty$ at finite temperature [21, 27]. As explained in Ref. [21], above the temperature at which the symmetry dynamically broken is fully restored, that is $M_{i}=m_{i}$, the condensates are set to zero. The use of the infinite cutoff allows the presence 


\begin{tabular}{||c||c|c|c|c|c||}
\hline Scenario B & $\begin{array}{c}T_{0} \\
{[\mathrm{MeV}]}\end{array}$ & $\begin{array}{c}T_{\chi} \\
{[\mathrm{MeV}]}\end{array}$ & $\begin{array}{c}T_{d} \\
{[\mathrm{MeV}]}\end{array}$ & $\begin{array}{c}\Delta \\
-\end{array}$ & $\begin{array}{c}T_{\text {eff }} \\
{[\mathrm{MeV}]}\end{array}$ \\
\hline \hline PNJL & 210 & 215 & 177 & $18 \%$ & $\sim 250$ \\
& 270 & 237 & 219 & $8 \%$ & $\sim 300$ \\
\hline EPNJL & 170 & 173 & 173 & - & $\sim 200$ \\
& 270 & 223 & 223 & - & $\sim 300$ \\
\hline
\end{tabular}

TABLE IV. Characteristic temperatures in the PNJL and the EPNJL model for different values of $T_{0}\left(\Delta=\left(T_{\chi}-T_{d}\right) / T_{\chi}\right)$.

of high momentum quarks, ensuring that the pressure goes to the Stefan-Boltzmann limit and, as shown in [32] gives a better description of several thermodynamic quantities.

As it can be seen in Table IV, in the PNJL model $T_{\chi}$ and $T_{d}$ never coincide but are closer for higher values of $T_{0}$, so it is adequate $T_{0} \simeq 270 \mathrm{MeV}$, the value used in the pure gauge approach. We also present results for $T_{0}=210 \mathrm{MeV}$, the value derived by RG considerations. The transition temperatures are defined by the peaks in the susceptibilities of the chiral condensate, for the restoration of chiral symmetry, and of the Polyakov loop, for the confinement-deconfinement transition.

However, in the EPNJL model, where $\Delta=\left(T_{\chi}-T_{d}\right) / T_{\chi}=0$ by construction, we have more freedom to fix $T_{0}$ and a lower value of $T_{0}$ is convenient $(\simeq 170 \mathrm{MeV})$ since it allows one to reproduce lattice results for the critical temperature for deconfinement and restoration of chiral symmetry [6]. The results are presented for scenario B, for reasons that will be explained latter.

In the EPNJL model the coupling constants are replaced by effective couplings dependent on $\Phi$, which ensures the entanglement between deconfinement and restoration of chiral symmetry. Now, using a EPNJL model with anomaly, a question arises: is the restoration of axial symmetry also entangled with two phase transitions mentioned above or should we have, like in PNJL model, an independent mechanism of instanton suppression? Having in mind the PNJL results [22], we will discuss results for the following cases that are summarized in Table III

- $\mathrm{G}(\Phi)$, with $g_{1}(\Phi), g_{2}(\Phi)$, without extra dependence on temperature. 

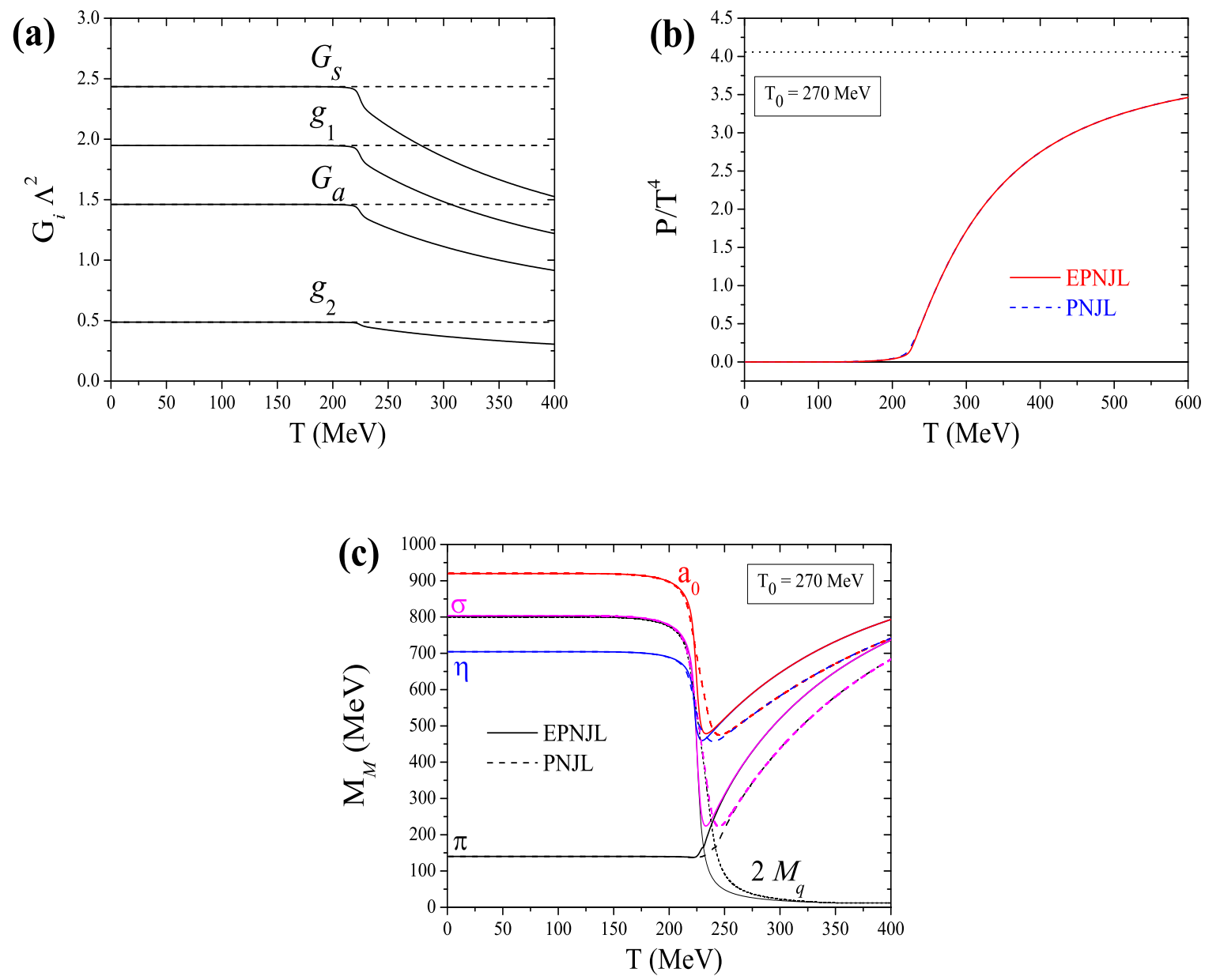

FIG. 1. Comparison of several quantities in the PNJL model (dashed lines) and the EPNJL model (full lines), without explicit dependence of $g_{1}$ and $g_{2}$ on temperature and at $T_{0}=270 \mathrm{MeV}$ : coupling vertices (a), pressure (b), and meson masses (c).

- $\mathrm{G}(\Phi)$, with $g_{1}(\Phi, T), g_{2}(\Phi, T)$, scenario $\mathrm{B}$, the explicit dependence on temperature being introduced, as in the PNJL model, through Eq. (20).

Let us concentrate on observables related with the restoration of axial symmetry. Concerning the topological susceptibility, its vanishing is guaranteed in both models since the infinite cutoff leads to the vanishing of the quark condensate and $\chi$ is proportional to $\langle\bar{q} q\rangle^{2}$ [see Eq. (13) $]$. As for the gap between the masses of the meson axial partners, in order to get its vanishing one should have $G_{a} \rightarrow G_{s}$. We begin by considering no explicit mechanism of instanton suppression in the models, that is: we have $g_{1}$ and $g_{2}$ constants, in the PNJL, 

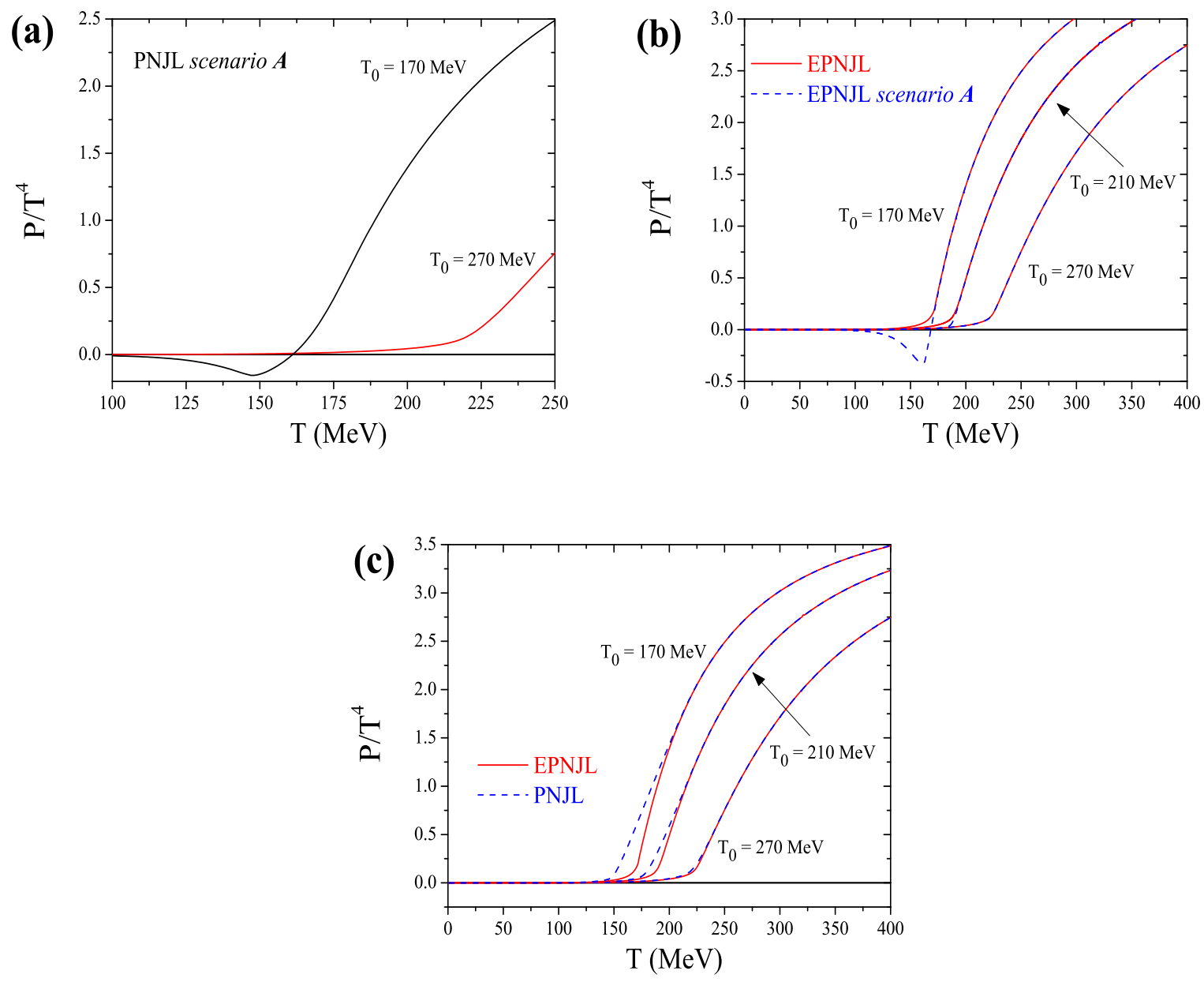

FIG. 2. Pressure for different values of $T_{0}$ with scenario $\mathrm{A}$ in PNJL and EPNJL models [panels (a) and (b)]; with scenario B in PNJL and EPNJL models [panel (c)].

and $g_{1}(\Phi)$ and $g_{2}(\Phi)$ in EPNJL [column I of Table III]. As it can be seen in Fig. 1(a) for $T_{0}=270 \mathrm{MeV}$, although in the EPNJL model the effective vertices have a temperature dependence through the Polyakov field, $\Phi, G_{s}$ does not converge to $G_{a}$ and, consequently, the axial partners $\left(\pi, a_{0}\right)$ and $(\sigma, \eta)$ do not degenerate in Fig. (1(c)). Therefore, we conclude that in both models we need an explicit temperature dependence of the coupling coefficients in order to have effective restoration of axial symmetry. Finally, we notice that the results for the pressure are qualitatively similar in both models [Fig. 11(b)].

In order to check whether the scenarios explored for the temperature dependence are physically meaningful, we begin by plotting in Fig. 2 the pressure for different scenarios. 
We see that in scenario A, for both models, there is an unphysical region of negative pressures for low values of $T_{0}$ [Figs. 2(a) 2(b)]. This is probably due to a too fast instanton suppression when $T_{0}$ takes lower values, so we need a mechanism to balance this effect if we want to consider low values of $T_{0}$. This problem does not exist in scenario B [Fig. 2(c)], and, as will be seen (Figs. 3 and 4), this ansatz also guarantees the restoration of axial symmetry. So, scenario $B$, which allows freedom in fixing $T_{0}$, will be the scenario adopted in the remainder of the present work. The difference in the behavior of the pressure in both scenarios can be understood from Eq. (17), which depends explicitly on the coupling constants through $G_{s}=g_{1}+g_{2}$. The coupling $G_{s}$ depends on $c(T)[$ Eq. (20)] in scenario A, but not in scenario B.

In Fig. 3] we plot the PNJL results for the meson masses, considering two values of $T_{0}$, in scenario B, and the topological susceptibility for different cases. We see that both the topological susceptibility as well as the gap between chiral and axial partners vanish, so axial symmetry is effectively restored. For $T_{0}=270 \mathrm{MeV}$ the convergence of chiral partners occurs before that of axial partners, as usual, with $T_{\text {eff }} \approx 300 \mathrm{MeV}\left(T_{\text {eff }}\right.$ is the temperature at which the effective restoration of the two symmetries is achieved). The new finding is that for $T_{0}=210 \mathrm{MeV}$ the chiral and axial partners get degenerate very closely and $T_{\text {eff }}$ is lower. An exploration of this temperature region could lead to a discussion about the sequence of restoration of two symmetries or even of its possible coincidence. Concerning the topological susceptibility [Fig. 3(c)], the behavior is quite similar for scenario A and B and it vanishes at $T \approx 325 \mathrm{MeV}$; when both $g_{1}$ and $g_{2}$ are kept constant, the topological susceptibility vanishes later, in this case this effect is only due to the vanishing of the quark condensate, a consequence of the full restoration of chiral symmetry.

The behavior of the meson masses and topological susceptibility in the EPNJL model is qualitatively similar to the PNJL model, as it can be seen in Fig. 4. Here, also the restoration of chiral and axial symmetries becomes closer for low values of $T_{0}$, but the temperatures for the effective restoration of symmetries are slightly lower. We conclude that in EPNJL there is entanglement between restoration of chiral symmetry and deconfinement but not with restoration of axial symmetry. In order to have restoration of this symmetry an explicit correlated dependence on $T$ of $g_{1}(\Phi)$ and $g_{2}(\Phi)$, which ensures instanton suppression and gives meaningful physical results, is necessary. 

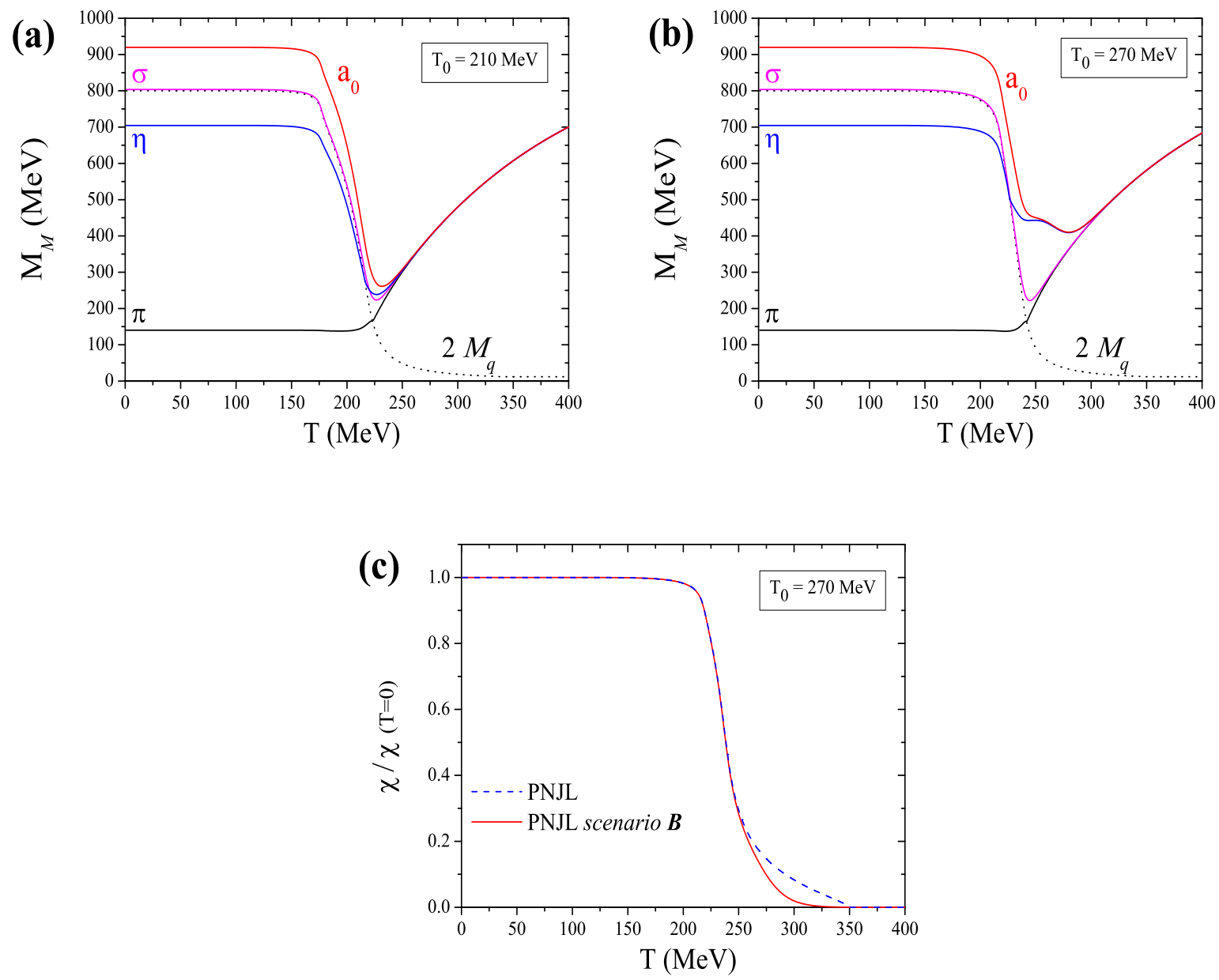

FIG. 3. Meson masses in the PNJL model with scenario B for two values of $T_{0}$ and topological susceptibility with different scenarios.

\section{CONCLUSIONS}

We performed a comparative study of deconfinement, restoration of chiral and axial symmetries within the framework of the PNJL and EPNJL models. In the EPNJL model the coupling coefficients are endowed with a dependence on the Polyakov field, $\Phi$, which guarantees that deconfinement and restoration of chiral symmetry occurs at the same temperature, which can be fitted to lattice result by choosing a low value for the parameter $T_{0}=170 \mathrm{MeV}$. In order to discuss the effective restoration of chiral and axial symmetries, we calculate the topological susceptibility and the masses of the mesons which are chiral and axial partners. At finite temperature we consider the cutoff $\Lambda \rightarrow \infty$, in order to get the Stefan-Boltzmann 

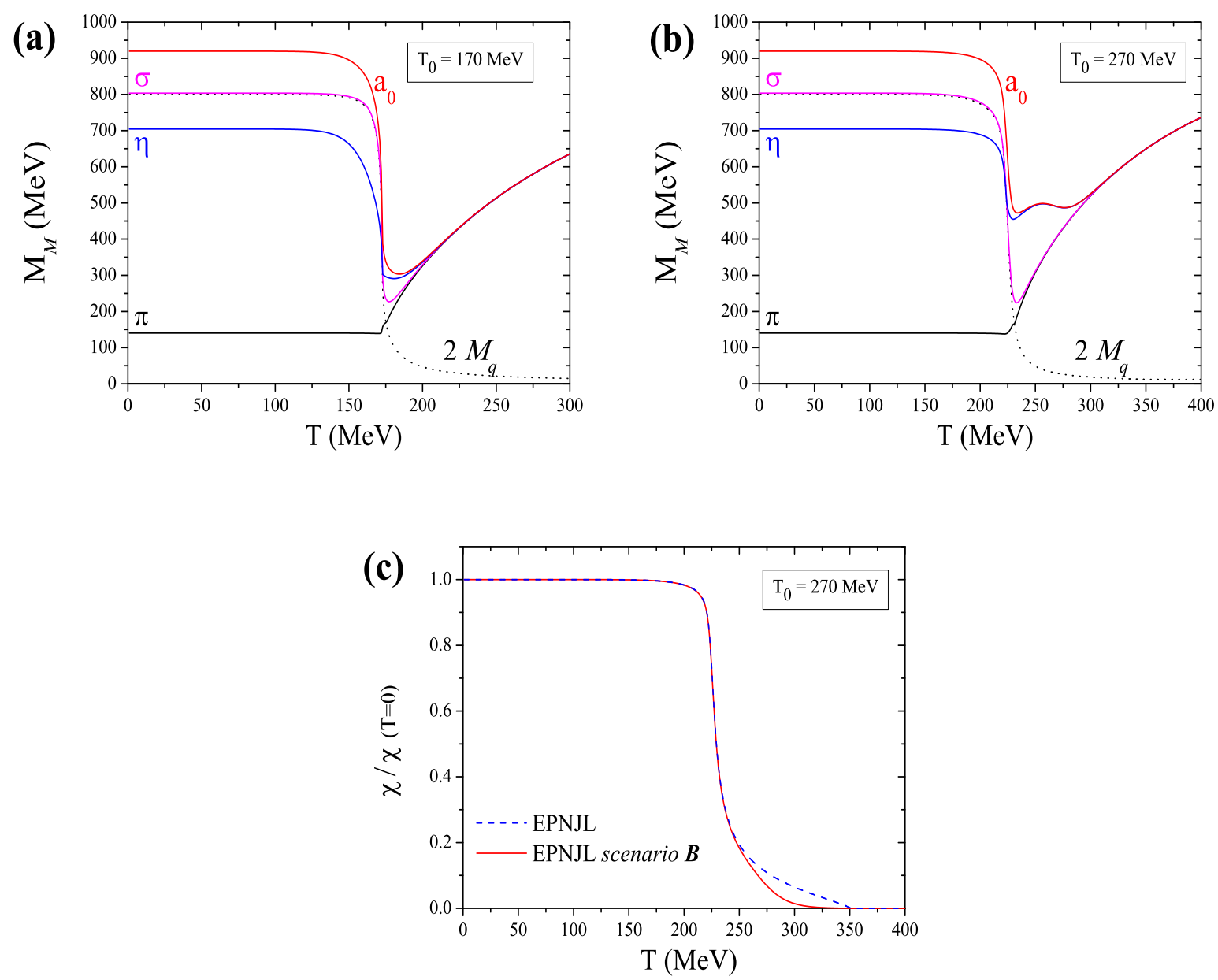

FIG. 4. Meson masses in the EPNJL model with scenario B for two values of $T_{0}$ [panels (a) and (b)] and topological susceptibility with two scenarios [panel (c)].

limit for the pressure.

In both models we verified that the masses of chiral partners $\sigma$ and $\pi$ converge and that the topological susceptibility vanishes, as a consequence of the effective restoration of chiral symmetry that occurs when the quark condensate vanishes. However, if no explicit temperature dependence of the coupling coefficient $g_{2}$ is considered, the masses of the axial partners do not converge and anomaly effects remain in the chirally symmetric vacuum. In order to get the vanishing of the anomaly it is enough that only $g_{2}$ is a decreasing function of the temperature that eventually goes to zero. However, if there is no mechanism to balance a fast decreasing of $g_{2}$, for low values of $T_{0}$, an unphysical region of negative pressures 
occurs. To prevent this, and have more freedom in the choice of $T_{0}$, we consider both $g_{1}$ and $g_{2}$ as explicit functions of temperature, $g_{2}$ going to zero while $g_{1}$ attains a maximum value. This approach also guarantees the convergence of the axial partners, $\left(\pi, a_{0}\right)$ and $(\sigma, \eta)$. In both models the restoration of both symmetries is closer when low values of $T_{0}$ are used. Our results follow the tendency of recent lattice results (calculated in the chiral limit), in particular in what concerns the degeneracy of mesons chiral and axial partners [9, 10, 12]. Finally, we remark that although in the EPNJL model there is entanglement between restoration of chiral symmetry and deconfinememt, the restoration of the axial symmetry needs an extra assumption that ensures the annihilation of the contribution of the 't Hooft interaction. It should be noticed that the critical temperature, $T_{\chi}$, signals only the partial restoration of chiral symmetry, its effective restoration occurring only at $T_{\text {eff }}$ when the chiral partners get degenerate. So, even when chiral and axial symmetry become effectively restored, since $T_{\text {eff }}>T_{\chi}$, some effects of the breaking of both symmetries remain above the critical temperature, in the interval $\left(T_{\chi}, T_{\text {eff }}\right)$.

\section{ACKNOWLEDGMENTS}

This work was supported by Projects No. CERN/FP/116356/2010 and No. PTDC/FIS/ 100968/2008, projects developed under the initiative QREN financed by the UE/FEDER through the Program COMPETE - "Programa Operacional Factores de Competitividade".

[1] P. N. Meisinger, and M. C. Ogilve, Phys. Lett. B 379, 163 (1996).

[2] K. Fukushima, Phys. Lett. B 591, 277 (2004).

[3] C. Ratti, M. A. Thaler, and W. Weise, Phys. Rev. D 73, 014019 (2006).

[4] E. Megias, E. Ruiz Arriola, and L.L. Salcedo, Phys. Rev. D 74, 065005 (2006); Phys. Rev. D 74, 114014 (2006).

[5] A. Bazavov et al. (HotQCD Collaboration), Phys. Rev. D 85, 054503(2012).

[6] V. Bornyakov et al. (QCDSF-DIK Collaboration), Phys. Rev. D 82, 014504 (2010); arXiv:1102.4461. 
[7] Y. Sakai, T. Sasaki, H. Kouno, and M. Yahiro, Phys. Rev. D 82, 076003 (2010); J. Phys. G: Nucl. Part. Phys. 39, 035004 (2012).

[8] H. Kouno, Y. Sakai, T. Sasaki, K. Kashiwa, and M. Yahiro, Phys. Rev. D 83, 076009 (2011).

[9] A. Bazavov, et al. (HotQCD Collaboration), Phys. Rev. D 86, 094503 (2012).

[10] S. Aoki, H. Fukaya, Y. Taniguchi arXiv:1209.2061 [hep-lat].

[11] B. Alles, M. D'Elia, and A. Di Giacomo, Nucl. Phys. B494, 281 (1997); B. Alles and M. D'Elia, arXiv:hep-lat/0602032.

[12] G. Cossu et al. (JLQCD Collaboration), Proc. Sci., LATTICE2011, 188 (2011), arXiv:1204.4519 [hep-lat]].

[13] T. Kunihiro, Phys. Lett. B 219 363(1989); T. Hatsuda and T Kunihiro, Phys. Rep. 247, 221 (1994).

[14] P. Costa, M. C. Ruivo and Yu.L. Kalinovsky, Phys. Lett. B 560, 171(2003); P. Costa, M. C. Ruivo, C.A. de Sousa, and Yu.L. Kalinovsky, Phys.Rev. C 70, 025204 (2004).

[15] P. Costa, M. C. Ruivo, C.A. de Sousa, and Yu.L. Kalinovsky, Phys. Rev. D 70, 116013 (2004); Phys. Rev. D 71, 116002 (2005).

[16] E. Witten, Nucl. Phys. B 156, 269 (1979); G. Veneziano, Nucl. Phys. 159, 213 (1979).

[17] T. Gsörgö, R. Vértesi and J. Sziklai, Phys. Rev. Lett. 105, 182301 (2010).

[18] K. Fukushima, K.Ohnishi, and K. Ohta, Phys. Rev. C 63, 045203 (2001).

[19] P. Costa, M. C. Ruivo, C. A. de Sousa, H. Hansen, and W. M. Alberico, Phys. Rev. D 79, 116003 (2009); P. Costa, M. C. Ruivo, C. A. de Sousa, and H. Hansen, Europhys. Lett. 86, 31001 (2009); Symmetry 2(3), 1338 (2010).

[20] Y. Sakai, H. Kouno, T. Sasaki, and M. Yahiro, Phys. Lett. B 705, 349 (2011); R. Gatto and M. Ruggieri, Phys. Rev. D 85, 054013 (2012).

[21] P. Costa, M. C. Ruivo, and C. A. de Sousa, Phys.Rev.D 77, 096009 (2008).

[22] M. C. Ruivo, M. Santos, P. Costa, and C. A. de Sousa, Phys. Rev. D 85, 036001 (2012).

[23] R. D. Pisarski, Phys. Rev. D 62, 111501(R) (2000); R. D. Pisarski, arXiv:hep-ph/0203271.

[24] O. Kaczmarek, F. Karsch, P. Petreczky, and F. Zantow, Phys. Lett. B 543, 41 (2002).

[25] O. Kaczmarek, Proc. Sci., CPOD07, 043 (2007), arXiv:0710.0498.

[26] H. Hansen, W. M. Alberico, A. Beraudo, A. Molinari, M. Nardi, and C. Ratti, Phys. Rev. D 75, 065004 (2007).

[27] C. Ratti, S. Rössner, M. A. Thaler and W. Weise, Eur. Phys. J. C 49, 213 (2007); S. Rössner, 
C. Ratti and W. Weise, Phys. Rev. D 75, 034007 (2007).

[28] M. Frank, M. Buballa, and M. Oertel, Phys. Lett. B 562, 221 (2003).

[29] T. Brauner, K. Fukushima, and Y. Hidaka, Phys. Rev. D 80, 074035 (2009); 81, 119904(E) (2010).

[30] B.-J. Schaefer, J. M. Pawlowski, and J. Wambach Phys. Rev. D 76, 074023 (2007).

[31] O. Philipsen, arXiv:1207.5999 [hep-lat].

[32] P. Costa, H. Hansen, M. C. Ruivo, and C. A. de Sousa Phys. Rev. D 81, 016007 (2010); M. C. Ruivo, P. Costa, C. A de Sousa, H. Hansen and W. Alberico, AIP Conf. Proc. 1257, 770 (2010) arXiv:1001.3072 [hep-ph]]. 